\title{
Versatility of fear-potentiated startle paradigms for assessing human conditioned fear extinction and return of fear
}

\section{Seth D. Norrholm ${ }^{1,2}$ *, Kemp M. Anderson ${ }^{1,2}$, Ilana W. Olin ${ }^{1,2}$, Tanja Jovanovic ${ }^{2}$, Cliffe Kwon ${ }^{1,2}$, Victor T. Warren ${ }^{1,2}$, Alexander McCarthy ${ }^{1,2}$, Lauren Bosshardt ${ }^{1,2}$, Justin Sabree ${ }^{1,3}$, Erica J. Duncan ${ }^{1,2}$, Barbara O. Rothbaum ${ }^{2}$ and Bekh Bradley ${ }^{1,2}$}

1 Trauma Recovery Program, Mental Health Service Line, Atlanta Veterans Affairs Medical Center, Decatur, GA, USA

${ }^{2}$ Department of Psychiatry and Behavioral Sciences, Emory University School of Medicine, Atlanta, GA, USA

${ }^{3}$ Department of Psychology, Oglethorpe University, Atlanta, GA, USA

\section{Edited by:}

David M. Diamond, University of South Florida, USA

Reviewed by:

Marie H. Monfils, University of Texas

at Austin, USA

Phillip R. Zoladz, Ohio Northern

University, USA

Scott L. Rauch, McLean Hospital, USA

${ }^{*}$ Correspondence:

Seth D. Norrholm, Mental Health

Service Line 116A, Atlanta Veterans

Affairs Medical Center, 1670

Clairmont Road, Suite 1E 113C,

Decatur, GA 30033, USA.

e-mail: snorrho@emory.edu;

seth.norrholm@va.gov
Fear conditioning methodologies have often been employed as testable models for assessing learned fear responses in individuals with anxiety disorders such as post-traumatic stress disorder (PTSD) and specific phobia. One frequently used paradigm is measurement of the acoustic startle reflex under conditions that mimic anxiogenic and fear-related conditions. For example, fear-potentiated startle is the relative increase in the frequency or magnitude of the acoustic startle reflex in the presence of a previously neutral cue (e.g., colored shape; termed the conditioned stimulus or $\mathrm{CS}+$ ) that has been repeatedly paired with an aversive unconditioned stimulus (e.g., airblast to the larynx). Our group has recently used fear-potentiated startle paradigms to demonstrate impaired fear extinction in civilian and combat populations with PTSD. In the current study, we examined the use of either auditory or visual CSs in a fear extinction protocol that we have validated and applied to human clinical conditions. This represents an important translational bridge in that numerous animal studies of fear extinction, upon which much of the human work is based, have employed the use of auditory CSs as opposed to visual CSs. Participants in both the auditory and visual groups displayed robust fear-potentiated startle to the CS+, clear discrimination between the reinforced CS+ and non-reinforced CS-, significant extinction to the previously reinforced $\mathrm{CS}+$, and marked spontaneous recovery. We discuss the current results as they relate to future investigations of PTSD-related impairments in fear processing in populations with diverse medical and psychiatric histories.

Keywords: fear conditioning, acoustic startle, auditory, extinction, discrimination, human

\section{INTRODUCTION}

Fear conditioning paradigms have often been employed as testable laboratory models for the fear-related symptoms of anxiety disorders such as post-traumatic stress disorder (PTSD; Friedman, 2000; Norrholm, 2011). A frequently used psychophysiological measure of conditioned fear is fear-potentiated startle, or the relative increase in the frequency or magnitude of the acoustic startle reflex in the presence of a previously neutral cue (e.g., colored shape; termed the conditioned stimulus or CS) that was paired with an aversive cue (e.g., airblast to the larynx; termed the unconditioned stimulus or US; Davis, 1986). Fear-potentiated startle is an attractive translational clinical research tool in that virtually the same methodologies can be used with animal or human subjects. Our research group previously developed and validated a fear-potentiated startle extinction protocol in psychiatrically healthy volunteers (e.g., Norrholm et al., 2006, 2008) and then used this protocol to detect fear extinction impairments in both civilian (Norrholm et al., 2010) and combat veteran (Norrholm, 2011) populations with PTSD.
The previously described fear-potentiated startle paradigms have the potential to be used as an objective measure of: (1) fear-related PTSD symptomatology, (2) vulnerability to developing PTSD after exposure to a traumatic event, and (3) as a pre-clinical platform for assessing the utility of potential cognitive enhancers (e.g., D-cycloserine) for the treatment of anxiety disorders (e.g., Ressler et al., 2004). As such, an important intermediate step is the optimization of these psychophysiological paradigms for effectively investigating the clinical questions to be asked. Traditionally, human studies of conditioned fear extinction have employed visual cues such as colored shapes or neutral computergenerated images as conditioned stimuli (CSs; e.g., Phelps et al., 2004; Norrholm et al., 2006; Alvarez et al., 2007; Milad et al., 2009). However, the large foundation of pre-clinical animal studies that have informed human fear extinction investigations have employed auditory CSs (e.g., Cain et al., 2001; Milad and Quirk, 2002; Morris et al., 2005; Monfils et al., 2009). There has been little use of auditory CSs in human fear conditioning and extinction studies to date. The primary objective of the current study was to investigate the use of auditory CSs in a previously established 
fear-potentiated startle extinction paradigm. For comparison, a contemporaneous group of psychiatrically healthy individuals was administered our previously used visual cue extinction paradigm as well.

\section{MATERIALS AND METHODS PARTICIPANTS}

Forty-three participants (20 females/23 males) with a mean age of $21(+0.3)$ years were enrolled in this study after signing an informed consent form approved by the Emory University Institutional Review Board, the Atlanta VAMC Research and Development Committee, and the US Army Medical Research and Materiel Command (USAMRMC)/Office of Research Protections (ORP)/Human Research Protection Office (HRPO). The psychiatrically healthy volunteers (Auditory protocol, $N=28$; Visual protocol, $N=15$ ) included in the current study were recruited as part of a larger investigation of fear extinction in combat veterans at the Atlanta VA Medical Center and were randomly assigned to the Auditory or Visual protocols. Inclusion requirements included corrected 20/20 vision without color blindness (assessed by eye chart and medical history review), tone detection at $30 \mathrm{~dB}[\mathrm{~A}] \mathrm{SPL}$ at frequencies ranging from 250 to $8000 \mathrm{~Hz}$ (assessed with a pure threshold audiometer, Grason-Stadler, Model GS1710), absence of current or past Axis I psychiatric diagnosis (assessed by Structured Clinical Interview for DSM-IV, SCID; First et al., 2002), and absence of significant trauma history (assessed by childhood trauma questionnaire, CTQ; Bernstein and Fink, 1998; SCID; and psychiatric history review). In addition, participants were screened for current illicit drug use by urine drug screen at the time of testing.

\section{ACOUSTIC STARTLE TESTING}

Trial Definitions: The eyeblink component of the acoustic startle response was measured according to previously published methods (Norrholm et al., 2006, 2011). The startle probe was a 108-dB [A], $40 \mathrm{~ms}$ burst of broadband white noise with near instantaneous rise time delivered binaurally with "earbud" headphones. Similar to several of our previous reports (e.g., Jovanovic et al., 2005; Norrholm et al., 2008), the aversive stimulus (US) was a $250 \mathrm{~ms}, 140$ p.s.i. airblast directed at the larynx. The CSs were either two distinct pure tones $(500$ or $4000 \mathrm{~Hz}$ pure tone; Auditory Cue protocol) or two colored geometric shapes (square or triangle; Visual Cue protocol) with stimulus reinforcement counterbalanced across participants. The two tones were matched for perceptual loudness in a pilot study according to the principles described by Fletcher and Munson (1933). CSs were presented to the participants for $6 \mathrm{~s}$ via binaural headphones (Auditory Cue protocol) or a computer screen positioned approximately $1 \mathrm{~m}$ from the participant (Visual Cue protocol). On CS+ trials, the tone or shape was presented for $6 \mathrm{~s}$ total, with the $40 \mathrm{~ms}$ startle probe presented $5210 \mathrm{~ms}$ after CS onset followed $500 \mathrm{~ms}$ later by the $250 \mathrm{~ms}, 140$ p.s.i. airblast that co-terminated with the CS presentation. On CS- trials, the tone or light was presented for $6 \mathrm{~s}$ total, with the startle probe occurring $5960 \mathrm{~ms}$ after CS onset. On noise alone (NA) trials, the $40 \mathrm{~ms}$ startle probe was presented alone, without the CSs. The Auditory and Visual protocols included only experimentally naïve participants, thus no participant was included in both sessions resulting in two separate cohorts.

\section{FEAR ACQUISITION, EXTINCTION TRAINING, AND EXTINCTION TEST}

The Fear Acquisition and Extinction Training sessions were administered on the first day of testing with a 10-min break between the two phases. During the 10-min break, participants completed a brief, emotionally neutral handedness survey. Twenty-four hours after the Extinction Training session, an Extinction Test (index of extinction recall or spontaneous recovery) was administered. All three sessions were conducted in the same context.

The Fear Acquisition session began with a 1-min acclimation period followed by a habituation phase consisting of three NA presentations. Next, a CS habituation phase was presented consisting of two presentations of each CS without the airblast US. After habituation to the CSs, the Fear Acquisition session continued with three blocks of four trials of each trial type (CS+, CS-, and NA). The Fear Acquisition session used a 100\% reinforcement schedule for the CS+. In other words, each CS+ presentation was paired with the airblast US. The intertrial interval (ITI) was randomized between 9 and $22 \mathrm{~s}$. The Extinction Training session occurred $10 \mathrm{~min}$ after Fear Acquisition and began with a habituation phase of three NA presentations. Next, the Extinction Training session continued with six blocks of four trials of each type (previously reinforced $\mathrm{CS}+, \mathrm{CS}-$, and NA) with no airblasts presented after any of the CS trials. The ITI was the same as above. One day after Extinction Training, participants were presented with an Extinction Test that began with nine NA presentations in an effort to reduce context conditioning (see Norrholm et al., 2008). The NA presentations were followed by one block consisting of four trials of each trial type (previously reinforced $\mathrm{CS}+, \mathrm{CS}-$, and NA) with no airblasts presented after any of the CS trials. Again, the ITI was between 9 and $22 \mathrm{~s}$.

As described previously, the current study represents an initial investigation of auditory CSs in a fear-potentiated startle extinction paradigm. As such, a modification of the previously published methods (e.g., Norrholm et al., 2010) was added. To prevent rapid habituation to the acoustic startle probe (due to the repeated auditory stimulation via acoustic startle probes and auditory CSs), a startle probe was included in only two of the four presentations of the CSs in each Block during the Fear Acquisition and Extinction Training phases. For consistency, the visual protocol included a startle probe in only two of the four presentations of the CSs in each Block during the Fear Acquisition and Extinction Training phases as well. All CS+ presentations during the Acquisition phases were paired with the airblast US for a $100 \%$ reinforcement schedule, regardless of whether or not an acoustic noise probe was included.

\section{DATA COLLECTION}

The acoustic startle response data were acquired using the electromyography (EMG) module of the BIOPAC MP150 (Biopac Systems, Inc., Aero Camino, CA, USA) according to our previously published methods (Jovanovic et al., 2010). The eyeblink component of the acoustic startle response was measured by EMG 
recordings of the right orbicularis oculi muscle (Norrholm et al., 2006, 2008; Jovanovic et al., 2009).

A response keypad (SuperLab, Cedrus Corp., San Pedro, CA, USA) was used during each acoustic startle session to record the participants' expectancy of the US on each CS trial (Jovanovic et al., 2006).

\section{DATA ANALYSIS}

Fear conditioning was first assessed by comparing startle magnitude in the presence of the CS+ to that of the NA trials. In order to examine differential conditioning and extinction, the degree of fear-potentiated startle was calculated using a Difference Score [(startle magnitude in the presence of a CS in each conditioning block) - startle magnitude to the noise probe alone (NA)]. For the Fear Acquisition phase, variables were analyzed in a Repeated Measures ANOVA with within-subject factors of Block (four levels) and trial type (two levels, CS+ and CS-) and a between-subjects factor of Session (Auditory or Visual). For the Extinction Training phase, variables were analyzed in a Repeated Measures ANOVA with within-subject factors of Block (six levels) and a betweensubjects factor of Session (Auditory or Visual). Lastly, for the Extinction Test, variables were analyzed in a Repeated Measures ANOVA with within-subject factors of Block (two levels; terminal block of extinction versus level of fear-potentiated startle at Test) and a between-subjects factor of Session (Auditory or Visual). Baseline startle was measured by comparing average startle magnitude to the noise probe alone during each test session. Contingency awareness was analyzed by comparing US-expectancy ratings of each CS trial with a Repeated Measures ANOVA with Trial number and Trial type as within-subjects factors and a between-subjects factor of Session (Auditory or Visual). All statistical analyses were performed in SPSS 19.0 for Windows (SPSS, Inc., Chicago, IL, USA), with $\alpha=0.05$.

\section{RESULTS \\ FEAR ACQUISITION PHASE}

Across all participants, pairing the CS+ with the aversive airblast US produced robust fear-potentiated startle upon presentation of the CS+ compared to startle to the noise probe alone (NA) during the Fear Acquisition session [Repeated Measures ANOVA, Significant Block $\times$ Trial Type interaction, $F(1,40)=21.7, p<0.001$ ] There was a significant between subjects main effect of Session $[F(1,40)=6.12, p<0.05]$ but no significant Block $\times$ Trial Type $\times$ Session interaction. In addition, across the entire Acquisition phase, all participants exhibited significant discrimination between the CS+ and CS- during Fear Acquisition as measured by acoustic startle [Repeated Measures ANOVA, Significant Block $\times$ Trial Type interaction, $F(1,40)=47.1, p<0.001$ ] with no significant Block $\times$ Trial Type $\times$ Session interaction or between subjects main effect (see Figures 1A,B). Acquisition of discrimination learning between the reinforced CS+ and non-reinforced CS- was evident as early as the first Block of the Acquisition phase in the Auditory session as compared to the Visual session [Repeated Measures ANOVA, Significant Trial Type $\times$ Session interaction, $F(1,40)=6.00, p<0.05$ ]. Follow-up comparison of Trial Type within each Block, indicated that there was significant CS discrimination on Block 1
$[F(1,26)=5.35, p=0.03]$, Block $2[F(1,26)=12.5, p=0.002]$, and Block $3[F(1,26)=33.5, p<0.001]$ of the Auditory protocol and Block $2[F(1,14)=20.1, p=0.001]$ and Block 3 $[F(1,14)=38.3, p<0.001]$ of the Visual protocol. Based on USexpectancy ratings on the response keypad, participants in both paradigms showed clear discrimination between the CS+ (Danger) and the CS- [Safety; Repeated Measures ANOVA, Significant Trial $\times$ Trial Type interaction, $F(1,29)=175.3, p<0.001$, Main Effect of Trial Type, $F(1,29)=470, p<0.001$, with no significant Trial $\times$ Trial Type $\times$ Session interaction or between subjects main effect; see Figures 1C,D].

\section{EXTINCTION TRAINING PHASE}

Participants underwent an Extinction Training phase 10 min after the conclusion of the Acquisition phase. All participants showed significant within-session extinction of fear-potentiated startle to the previously reinforced CS + [Repeated Measures ANOVA, Significant Main Effect of Block, $F(1,40)=78.6, p<0.001$, with a Significant Block $\times$ Session interaction $F(1,40)=4.63, p<05$; see Figure 2A]. Participants in the Auditory condition displayed a "steeper" slope of extinction that was due, in part, to a higher level of fear-potentiated startle at the beginning stages of the Extinction phase. Participants also exhibited a significant decrease in their US-expectancy ratings when presented with the previously reinforced CS+ during the Extinction Training phase [Repeated Measures ANOVA, Significant Main Effect of Trial, $F(1,37)=62.5, p<0.001$, with no significant Trial $\times$ Session interaction or between subjects main effect of Session; see Figure 2B].

\section{EXTINCTION TEST}

When presented with an Extinction Test $24 \mathrm{~h}$ after Extinction Training, participants displayed a significant return of fearpotentiated startle through spontaneous recovery regardless of CS modality [Repeated Measures ANOVA, Main Effect of Block, $F(1,34)=13.4, p=0.001$, with no significant Block $\times$ Session interaction or between subjects main effect of Session; see Figure 2A]. In addition, participant US-expectancy ratings increased during the Extinction Test as compared to the conclusion of the Extinction Training phase regardless of CS modality [Repeated Measures ANOVA, Main Effect of Trial, $F(1,31)=30.8$, $p<0.001$, with no significant Trial $\times$ Session interaction or between subjects main effect of Session; see Figure 2B].

\section{DISCUSSION}

The results of the current study demonstrate that the use of auditory or visual CSs produce robust fear-potentiated startle, clear discrimination between the reinforced CS+ and non-reinforced CS-, significant within-session extinction of fear-potentiated startle, and marked levels of spontaneous recovery of fear-potentiated startle. This study independently replicates what we have reported previously with visual CSs, validates the use of auditory CSs in human fear conditioning studies, and furthers the development and utilization of fear-potentiated startle paradigms for assessing conditioned fear extinction in humans.

These data have potential implications for future investigations in traumatized populations with PTSD in that the increased versatility of employing visual or auditory CSs will afford the 
A Auditory CSs

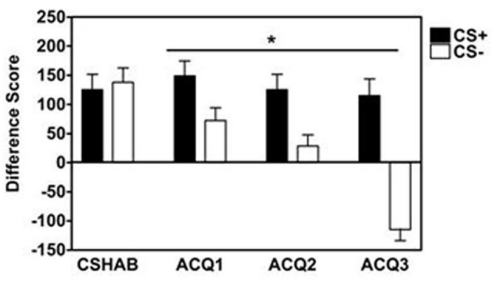

C Auditory CSs

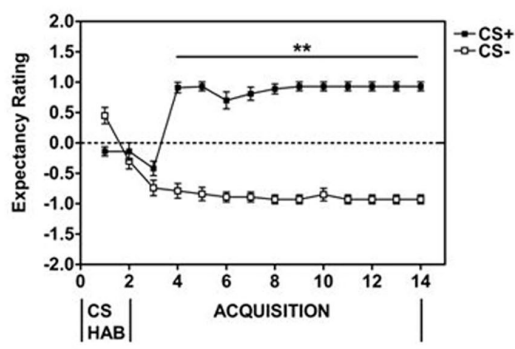

FIGURE 1 | Using fear-potentiated startle measures, psychiatrically healthy populations display clear discrimination between the CS+ and CS- during Fear Acquisition when employing either (A) Auditory or (B) Visual conditioned stimuli (CSs). Based on online US-expectancy measures, healthy volunteers also cognitively discriminated between the

\section{B Visual CSs}

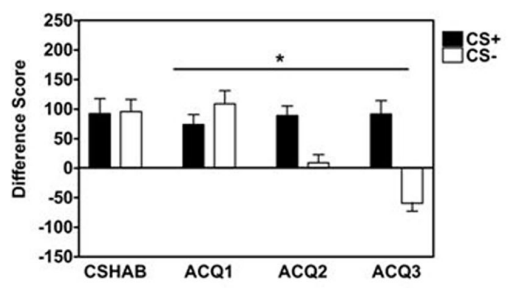

D Visual CSs

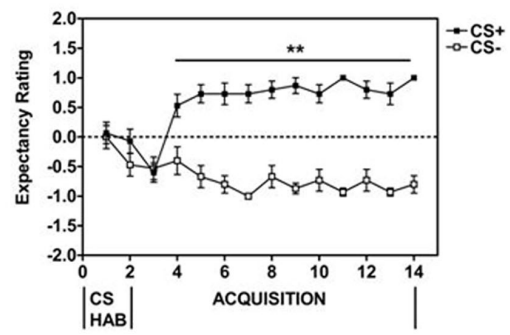

CS+ and CS - in the (C) Auditory and (D) Visual CS paradigms. Difference Score $=$ startle magnitude to a CS - startle magnitude to the noise probe alone (NA). CSHAB = CS habituation phase; $A C Q=$ acquisition block. *Significant Block $\times$ Trial Type interaction $(p<0.001)$; ${ }^{* *}$ significant Trial $\times$ Trial Type interaction $(p<0.001)$.

\section{A Fear Extinction - Fear-potentiated Startle}

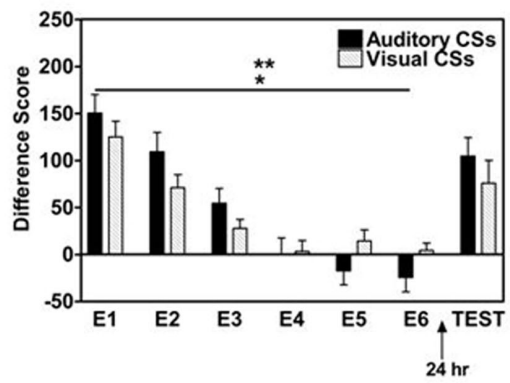

FIGURE 2 | (A) Using fear-potentiated startle measures,

psychiatrically healthy populations display significant within-session extinction to the previously reinforced CS+ during Extinction Training when employing either Auditory (dark bars) or Visual (hatched bars) conditioned stimuli. (B) Based on online US-expectancy measures, healthy volunteers showed a reduction in ratings of Danger on presentations of the previously reinforced

\section{B Fear Extinction - Cognitive Awareness}

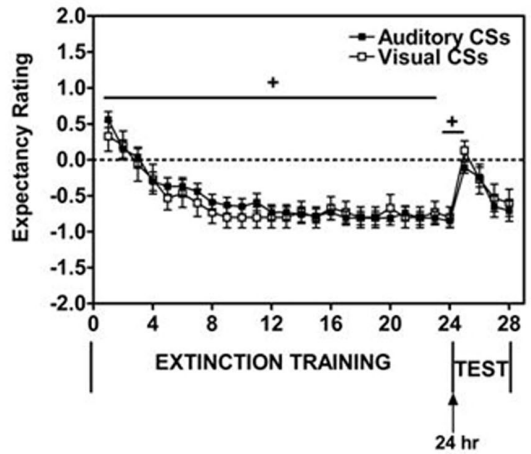

$\mathrm{CS}+$ Auditory (dark squares) and Visual (open squares) paradigms. Difference Score $=$ startle magnitude to a CS - startle magnitude to the noise probe alone (NA). E = extinction block. *Significant main effect of Block $(p<0.001)$; ${ }^{* *}$ significant Block $\times$ Session interaction $(p<0.05)$; + significant main effect of Trial $(p<0.001)$.

burns, motor vehicle accidents, or blunt force trauma injuries (Mader et al., 2006; Thach et al., 2008). Given that there is a high incidence of PTSD in veterans exposed to IEDs (Hoge, 2010), it appears likely that individuals with severe eye injuries will have concurrent PTSD symptoms and, as such, 
would benefit from increased study of fear dysregulation in this population.

In addition, the use of either auditory or visual CSs may prove beneficial in objectively studying treatment outcome in anxiety disorder patients who undergo extinction-based exposure therapy. For example, it may be possible to use a "cross-over" design in assessing the ability to extinguish learned fear before and after prolonged exposure treatment with the visual and auditory paradigms counterbalanced across subjects. The use of CSs of different sensory modalities may minimize the contribution of practice effects were participants administered the same paradigm at different time points.

In the current study, we employed auditory and visual fear conditioning paradigms that were based on our previously published studies of psychiatrically healthy volunteers and PTSD patients. Over the past several years, we have optimized this paradigm to include an Acquisition phase followed 10 min later by an Extinction phase that is then followed $24 \mathrm{~h}$ later by an Extinction Test. This design has proven effective in detecting extinction impairments in patient populations and as a reliable index of the return of fear. A significant advantage for patient populations is that several aspects of fear processing can be examined with a relatively small time commitment on the part of the participant. The development of a novel test design and validation of an auditory derivative of the existing paradigm will open up greater avenues for investigating the complexity of impaired fear and extinction learning in traumatized populations.

The results of the present study suggest that auditory stimuli may provide greater levels of fear conditioning and differences in extinction rate; differences that could potentially rule out the interchangeability of these protocols. A significant body of animal literature suggests that fear conditioning and fear extinction to visual and auditory CSs may be mediated by divergent neural pathways. While the medial prefrontal cortex has been widely implicated in the inhibition of conditioned fear memories (i.e., fear extinction; see Quirk and Mueller, 2008, there is evidence showing that sensory cortices are involved in fear acquisition and extinction to visual (Ledoux et al., 1989) and auditory (Romanski and Ledoux, 1992; Boatman and Kim, 2006; Song et al., 2010) CSs. For example, Song et al. (2010) replicated earlier work by Romanski and Ledoux (1992) by showing that auditory cortex lesions impair extinction to a tone CS previously paired with footshock. In addition, considerable differences in the neural circuitry underlying visual and auditory sensory processing may contribute to dissimilar levels of fear conditioning and extinction. For example, rodents develop conditioned fear responses to auditory cues at a faster rate than visual cues (Fendt and Fanselow, 1999; Ledoux,

\section{REFERENCES}

Alvarez, R. P., Johnson, L., and Grillon, C. (2007). Contextual-specificity of short-delay extinction in humans: renewal of fear-potentiated startle in a virtual environment. Learn. Mem. 14, 247-253.

Bernstein, D. P., and Fink, L. (1998). Childhood Trauma Questionnaire A Retrospective Self-Report Manual.
San Antonio, TX: The Psychological Corporation.

Boatman, J. A., and Kim, J. J. (2006). A thalamo-cortico-amygdala pathway mediates auditory fear conditioning in the intact brain. Eur. J. Neurosci. 24, 894-900.

Cain, C. K., Blouin, A. M., and Barad, M. (2001). L-type voltage gated calcium channels are

2000). The data from the current study showed a more rapid acquisition of $\mathrm{CS}+/ \mathrm{CS}-$ discrimination and more robust extinction in the auditory CS condition as compared to the visual CS condition. Further, conditioning to auditory cues is thought to be primarily mediated by a direct thalamo-amygdala pathway from the medial geniculate nucleus (MGN) and posterior intralaminar nucleus to the lateral amygdala (Rogan and Ledoux, 1995; Doron and Ledoux, 1999; Ledoux, 2000). Conversely, conditioning to visual cues is thought to be mediated by a more indirect thalamo-corticoamygdala pathway from the lateral geniculate nucleus (LGN) and lateral posterior thalamic nucleus to primary visual cortex to visual association area TE2/perirhinal cortex to the amygdala (Shi and Davis, 2001).

The current report provides an exciting new avenue for translational studies of fear extinction in patient populations. The primary purpose of the present work was to demonstrate the utility and validity of using either type of extinction protocol for detecting dysregulation in fear processing. The use of auditory cue paradigms increases accessibility of translational studies of fear and anxiety to patient populations with visual impairments and provides a potential platform for exploring clinical manipulations that may enhance extinction of fear maintained by subcortical thalamic amygdalar, as opposed to prefrontal cortical, pathways; this is especially relevant to anxiety patient populations that display a persistence of fear despite prolonged exposure therapy.

\section{ACKNOWLEDGMENTS}

Dr. Norrholm has research support from the National Alliance for Research on Schizophrenia and Depression (NARSAD), the Department of Defense (DoD)/Congressionally Directed Medical Research Program (CDMRP; \#W81XWH-08-2-0170), the Emory University Research Committee, and the PHS Grant (UL1 RR025008) from the Clinical and Translational Science Award Program, National Institutes of Health, National Center for Research Resources. Mr. Anderson, Ms. Olin, Mr. Kwon, Mr. Warren, Mr. McCarthy, Ms. Bosshardt, and Mr. Sabree report no financial interests, disclosures, or conflicts. Dr. Jovanovic has research support from NIMH (MH092576), NARSAD, and the Emory Care Medical Foundation. Dr. Duncan has research support from the National Institute on Drug Abuse (1R01DA018294-01A2) and the VA Merit Review Program. Dr. Rothbaum has funding from Department of Defense Clinical Trial Grant No. W81XWH-101-1045, NIMH, Grant No. U19 MH069056-03, NIMH Grant No. R34 MH 083078-01A1, and NIMH Grant No. 1R01MH70880-01A2. Dr. Bradley has research support from the VA Merit Review Program.

required for extinction, but not for acquisition or expression of conditional fear in mice. J. Neurosci. 22, 9113-9121.

Davis, M. (1986). Pharmacological and anatomical analysis of fear conditioning using the fear-potentiated startle paradigm. Behav. Neurosci. 100 814-824.
Doron, N. N., and Ledoux, J. E. (1999). Organization of projections to the lateral amygdala from auditory and visual areas of the thalamus in rats. J. Comp. Neurol. 384, 59-70.

Fendt, M., and Fanselow, M. S. (1999). The neuroanatomical and neurochemical basis of conditioned fear. Neurosci. Biobehav. Rev. 23, 743-760. 
First, M. B., Spitzer, R. L., Gibbon, M., and Williams, J. B. W. (2002). Structured Clinical Interview for DSMIV-TR Axis I Disorders, Research Version, Patient Edition (SCID-I/P). New York: New York State Psychiatric Institute.

Fletcher, H., and Munson, W. A. (1933). Loudness, its definition, measurement and calculation. J. Acoust. Soc. Am. 5, 82-108.

Friedman, M. J. (2000). What might the psychobiology of posttraumatic stress disorder teach us about future approaches to pharmacotherapy? J. Clin. Psychiatry 61, 44-51.

Hoge, C. W. (2010). Once a Warrior Always a Warrior: Navigating the Transition from Combat to Home, Including Combat Stress, PTSD and $m$ TBI. Guilford, CT: Globe Pequot Press.

Jovanovic, T., Keyes, M., Fiallos, A., Myers, K. M., Davis, M., and Duncan, E. (2005). Fear potentiation and fear inhibition in a human fearpotentiated startle paradigm. Biol. Psychiatry 57, 1559-1564.

Jovanovic, T., Norrholm, S. D., Blanding, N. Q., Phifer, J. E., Weiss, T., Davis, M., Duncan, E., Bradley, B., and Ressler, K. (2010). Fear potentiation is associated with hypothalamic-pituitary-adrenal axis function in PTSD. Psychoneuroendocrinology 35, 846-857.

Jovanovic, T., Norrholm, S. D., Fennell, J. E., Keyes, M., Fiallos, A., Myers, K. M., Davis, M., and Duncan, E. J. (2009). Posttraumatic stress disorder may be associated with impaired fear inhibition: relation to symptom severity. Psychiatry Res. 167, 151-160.

Jovanovic, T., Norrholm, S. D., Keyes, M., Fiallos, A., Jovanovic, S., Myers, K. M., Davis, M., and Duncan, E. J. (2006). Contingency awareness and fear inhibition in a human fearpotentiated startle paradigm. Behav. Neurosci. 120, 995-1004.
Ledoux, J. E. (2000). Emotion circuits in the brain. Annu. Rev. Neurosci. 23 155-184.

Ledoux, J. E., Romanski, L., and Xagoraris, A. (1989). Indelibility of subcortical memories. J. Cogn. Neurosci. 1, 238-243.

Mader, T. H., Carroll, R. D., Slade, C. S., George, R. K., Ritchey, J. P., and Neville, S. P. (2006). Ocular war injuries of the Iraqi insurgency, January-September 2004. Opthalmology 113, 97-104.

Milad, M. G., and Quirk, G. J. (2002). Neurons in medial prefrontal cortex signal memory for fear extinction. Nature 420, 70-74.

Milad, M. R., Pitman, R. K., Ellis, C. B., Gold, A. L., Shin, L. M., Lasko, N. B., Zeidan, M. A., Handwerger, K., Orr, S. P., and Rauch, S. L. (2009). Neurobiological basis of failure to recall extinction memory in posttraumatic stress disorder. Biol. Psychiatry 66, 1075-1082.

Monfils, M.-H., Cowansage, K. K., Klann, E., and Ledoux, J. E. (2009). Extinction-reconsolidation boundaries: key to persistent attenuation of fear memories. Science 324, 951-955.

Morris, R., Westbrook, R., and Killcross, A. (2005). Reinstatement of extinguished fear by betaandrenergic arousal elicited by a conditional context. Behav. Neurosci. 119, 1662-1671.

Norrholm, S. (2011). Translational fear inhibition models as indicies of trauma-related psychopathology. Curr. Psychiatry Rev. 7, 194-204.

Norrholm, S. D., Jovanovic, T., Olin, I. W., Sands, L., Karapanou, I., Bradley, B., and Ressler, K. J. (2010). Fear extinction in traumatized civilians with posttraumatic stress disorder: relation to symptom severity. Biol. Psychiatry 69, 556-563.

Norrholm, S. D., Jovanovic, T., Olin, I. W., Sands, L. A., Karapanou, I., Bradley, B., and Ressler, K. J. (2011).
Fear extinction in traumatized civilians with posttraumatic stress disorder: relation to symptom severity. Biol. Psychiatry 69, 556-563.

Norrholm, S. D., Jovanovic, T., Vervliet, B., Myers, K. M., Davis, M., Rothbaum, B. O., and Duncan, E. J. (2006). Conditioned fear extinction and reinstatement in a human fearpotentiated startle paradigm. Learn. Mem. 13, 681-685.

Norrholm, S. D., Vervliet, B., Jovanovic, T., Boshoven, W., Myers, K. M., Davis, M., Rothbaum, B. O., and Duncan, E. J. (2008). Timing of extinction relative to acquisition: a parametric analysis of fear extinction in humans. Behav. Neurosci. 122, 1016-1030.

Phelps, E. A., Delgado, M. R., Nearing, K. I., and Ledoux, J. E. (2004). Extinction learning in humans: role of the amygdala and vmPFC. Neuron 43, 897-905.

Quirk, G. J., and Mueller, D. (2008). Neural mechanisms of extinction learning and retrieval. Neuropsychopharmacology 33, 56-72.

Ressler, K. J., Rothbaum, B. O., Tannenbaum, L., Anderson, P., Graap, K., Zimand, E., Hodges, L., and Davis, M. (2004). Cognitive enhancers as adjuncts to psychotherapy: use of $\mathrm{D}$-cycloserine in phobic individuals to facilitate extinction of fear. Arch. Gen. Psychiatry 61, 1136-1144.

Rogan, M. T., and Ledoux, J. E. (1995). LTP is accompanied by commensurate enhancement of auditoryevoked responses in a fear conditioning circuit. Neuron 15, 127-136.

Romanski, L. M., and Ledoux, J. E. (1992). Equipotentiality of thalamo-amygdala and thalamocortico amygdala circuits in auditory fear conditioning. J. Neurosci. 12, 4501-4509.

Shi, C., and Davis, M. (2001). Visual pathways involved in fear conditioning measured with fearpotentiated startle: behavioral and anatomic studies. J. Neurosci. 21, 9844-9855.

Song, E. Y., Boatman, J. A., Jung, M. W., and Kim, J. J. (2010). Auditory cortex is important in the extinction of two different tone-based conditioned fear memories in rats. Front. Behav. Neurosci. 4, 1-7.

Thach, A. B., Johnson, A. J., Carroll, R. B., Huchun, A., Ainbinder, D. J., Stutzman, R. D., Blaydon, S. M., Demartelaere, S. L., Mader, T. H., Slade, C. S., George, R. K., Ritchey, J. P., Barnes, S. D., and Fannin, L. A. (2008). Severe eye injuries in the war in Iraq, 2003-2005. Ophthalmology $115,377-382$.

Conflict of Interest Statement: The authors declare that the research was conducted in the absence of any commercial or financial relationships that could be construed as a potential conflict of interest.

Received: 16 August 2011; accepted: 31 October 2011; published online: 21 November 2011.

Citation: Norrholm SD, Anderson KM, Olin IW, Jovanovic T, Kwon C, Warren VT, McCarthy A, Bosshardt L, Sabree J, Duncan EJ, Rothbaum BO and Bradley $B$ (2011) Versatility of fear-potentiated startle paradigms for assessing human conditioned fear extinction and return of fear. Front. Behav. Neurosci. 5:77. doi: 10.3389/fnbeh.2011.00077

Copyright (c) 2011 Norrholm, Anderson, Olin, Jovanovic, Kwon, Warren, McCarthy, Bosshardt, Sabree, Duncan, Rothbaum and Bradley. This is an openaccess article subject to a non-exclusive license between the authors and Frontiers Media SA, which permits use, distribution and reproduction in other forums, provided the original authors and source are credited and other Frontiers conditions are complied with. 